\title{
Measurement of the Nucleon Nucleon Scattering Length with the ESC04 Interaction
}

\author{
Roberto Arceo*, Gerardo Jesús Escalera Santos, Orlando Díaz-Hernández \\ Centro de Estudios en Física y Matemáticas Básicas y Aplicadas, \\ Universidad Autónoma de Chiapas, Tuxtla Gutiérrez, México \\ Email: ${ }^{*}$ roberto.arceo@unach.mx
}

Received October 11, 2013; revised October 19, 2013; accepted November 6, 2013

Copyright (c) 2014 Roberto Arceo et al. This is an open access article distributed under the Creative Commons Attribution License, which permits unrestricted use, distribution, and reproduction in any medium, provided the original work is properly cited. In accordance of the Creative Commons Attribution License all Copyrights (c) 2014 are reserved for SCIRP and the owner of the intellectual property Roberto Arceo et al. All Copyright (C) 2014 are guarded by law and by SCIRP as a guardian.

\begin{abstract}
We have determined a value for the ${ }^{1} S_{0}$ neutron-neutron scattering length $\left(a_{n n}\right)$. The scattering length result is presented for the extended-soft-core (ESC04) interaction. The value obtained in the present work is $a_{n n}=$ $-18.6249 \mathrm{fm}$. The method of solution of the radial Schrödinger equation with nonlocal potential for nucleonnucleon pairs is described and the result is consistent with previous determinations of $a_{n n}=-18.63 \pm 0.10$ (statistical) $\pm \mathbf{0 . 4 4}$ (systematic) $\pm \mathbf{0 . 3 0}$ (theoretical) $\mathrm{fm}$. The nonlocal potentials are of the central, spin-spin, spin-orbital, and tensor type. The analysis from the ESC04 interaction is done at energies $0 \leq T_{\text {lab }} \leq 350 \mathrm{MeV}$. We compare the present result with experimental S-wave phase shifts analysis and agreement is found.
\end{abstract}

\section{KEYWORDS}

\section{Nucleon-Induced Reactions; S-Matrix Theory; Scattering Theory}

\section{Introduction}

In nuclear physics, important information can be obtained from the scattering length associated with lowenergy nucleon-nucleon scattering. At these energies, the nucleon-nucleon interaction can be treated non-relativistically and the scattering was studied by means of a single particle Schrödinger equation which involves a nonlocal effective potential, derived from [1-4] using an extended soft-core model (ESC interaction). In the present manuscript, we consider a potential that involves a central part, a spin-spin interaction, a spin-orbital interaction and a tensor part and perform a numerical study of the associated Schrödinger equation. Also, we determine a numerical value for proton-proton and neutron-proton scattering lengths.

The present work is realized by considering energies in the range of $0 \leq T_{\text {lab }} \leq 350 \mathrm{MeV}$. For nucleon-nucleon scattering, it has been demonstrated that the interaction from the ESC model gives a description that is in good agreement with the nucleon-nucleon data. The extended

\footnotetext{
"Corresponding author.
}

soft-core model, also known as ESC, is used for nucleonnucleon (NN), hyperon-nucleon ( $\mathrm{YN})$, and hyperonhyperon (YY) scatterings. The particular version of the model ESC, called ESC04 [T. A. Rijken, Phys. Rev. C 73, 04007 (2006)], describes NN and YN interaction in an unified way using broken SU (3) symmetry.

A good fit with the experimental data is obtained by using the ESC04 model. The manuscript is organized as follows: in Section II, we give a theoretical review of the model; in Section III, we present our numerical results and in Section IV, we draw our conclusions.

\section{Theory}

\subsection{The Schroedinger Equation with Non-Local Potential}

The model we are going to study numerically involves a radial Schrödinger equation with ESC04 potential; namely

$$
\left[-\frac{\hbar^{2}}{2 \mu} \nabla^{2}+V(r)\right] \Psi(\vec{r})=E \Psi(\vec{r}),
$$


where $\mu=\frac{m_{1} m_{2}}{m_{1}+m_{2}}$ is the reduced mass of the nucleons whose individual masses are $m_{1}$ and $m_{2}$, and have spins $\overrightarrow{\sigma_{1}}$ and $\overrightarrow{\sigma_{2}} ; r$ is the distance between the nucleons. The potential is parameterized as

$$
V(r)=V_{c}(r)+V_{S S}(r) \vec{S} \bullet \vec{S}+V_{L S}(r) \vec{L} \bullet \vec{S}+S_{12} V_{T}(r)
$$

where $S_{12}=3\left(\overrightarrow{\sigma_{1}} \bullet \vec{r}\right)\left(\overrightarrow{\sigma_{2}} \bullet \vec{r}\right)-\left(\overrightarrow{\sigma_{1}} \bullet \overrightarrow{\sigma_{2}}\right)$ is a second rank tensor operator.

For an S-state we introduce $u(r)$, where

$$
\Psi(\vec{r})=\Psi(r)=\frac{u(r)}{r} .
$$

For a given value of the quantum number $J$,

$$
\Psi(\vec{r})=\sum_{L} \frac{u_{L}(r)}{r} \Phi_{J M L},
$$

where we introduce

$$
\Phi_{J M L}=\sum_{M_{L}=-L}^{L}\left(L 1 J \mid M_{L} M_{S} M\right) Y_{L M_{L}} \chi_{M_{S}},
$$

where the symbol $\left(L 1 J \mid M_{L} M_{S} M\right)$ denotes a ClebschGordan coefficient, and $Y_{\text {LML }}$ are the spherical harmonics, and

$$
\begin{gathered}
\chi_{+1}=\alpha_{1} \alpha_{2} ; \\
\chi_{0}=\frac{1}{\sqrt{2}}\left(\alpha_{1} \beta_{2}+\alpha_{2} \beta_{1}\right) ; \\
\chi_{-1}=\beta_{1} \beta_{2} .
\end{gathered}
$$

The subscript on $\chi$ refers to the magnetic projection quantum number $M_{S}$ of the spin- 1 state, while $\alpha$ and $\beta$ represent spin up and spin down for the particular spin- $-1 / 2$ nucleon indicated by the subscript.

The Equation (2) forms an orthonormal set spanning the space of spin-1 functions and functions of the direction $\boldsymbol{r}$. The normalization of $\Psi(r)$ requires that the radial functions satisfy,

$$
\sum_{L} \int_{0}^{\infty} u_{L}^{2}(r) d r=1 .
$$

The Schrödinger equation [Equation (1)] is processed by the method of separation of variables, we obtain as its radial component,

$$
\frac{d^{2}}{d r^{2}} R(r)+\frac{2}{r} \frac{d}{d r} R(r)+\left[\frac{2 \mu}{\hbar^{2}}\{E-V(r)\}-\frac{L(L+1)}{r^{2}}\right] R(r)=0 .
$$

We use the parametrized potential

$$
V(r)=V_{c}(r)+V_{S S}(r) \vec{S} \bullet \vec{S}+V_{L S}(r) \vec{L} \bullet \vec{S}+S_{12} V_{T}(r)
$$

and

for an S-state to obtain,

$$
R(r)=\frac{u(r)}{r}
$$

$$
\begin{aligned}
& \frac{d^{2}}{d r^{2}} u_{L}(r)+\frac{2 \mu}{\hbar^{2}}\left\{E-\frac{\hbar^{2}}{2 \mu r^{2}} L(L+1)-V_{c}(r)-S(S+1) V_{S S}(r)-\frac{1}{2} V_{L S}(r)[J(J+1)-L(L+1)-S(S+1)]\right\} u_{L}, \\
& -\frac{2 \mu}{\hbar^{2}} V_{T}(r) \sum_{L^{\prime}} S_{J L L^{\prime}} u_{L^{\prime}}(r)=0
\end{aligned}
$$

where $S_{J L L^{\prime}}=\int\left(\Phi_{J M L^{\prime}}, S_{12} \Phi_{J M L^{\prime}}\right) d \vec{r} \quad$ [5], and $S_{12}$ may be written as an operator of the form

$$
\sum_{q q^{\prime}}\left(j_{1} j_{2} \lambda \mid q q^{\prime} M\right) \overrightarrow{\sigma_{1 q}} \overrightarrow{\sigma_{2 q^{\prime}}}
$$

with $\lambda=2$ and $j_{1}=j_{2}=1$. Here $\left(j_{1} j_{2} \lambda \mid q q^{\prime} M\right)$ is the Clebsch-Gordan coefficient.

Using Racha algebra (see appendix A of [6]) we can show that

$$
S_{J L L^{\prime}}=(2 \sqrt{6}) L L^{\prime} \delta_{J J^{\prime}}(-1)^{1+J}\left(L L^{\prime} 2 \mid 000\right) W\left(L L^{\prime} 11 ; 2 J\right)=2 \delta_{J J^{\prime}}\left[\delta_{L L^{\prime}}-3(J 1 L \mid 000)\left(J 1 L^{\prime} \mid 000\right)\right] .
$$

\subsection{Numerical Solution of the Schrödinger Equation}

Considering the single state for the ${ }^{1} S_{0}$ wave, Equation (6) for the neutron-neutron system has the form $(S=J=L=0$, $\left.L^{\prime}=-1,0,1\right)$, 


$$
\begin{aligned}
& \frac{d^{2}}{d r^{2}} u_{0}(r)+\frac{2 \mu}{\hbar^{2}}\left\{E-V_{c}(r)\right\} u_{0}(r) \\
& -\frac{2 \mu}{\hbar^{2}} V_{T}(r)\left[S_{00-1} u_{-1}(r)+S_{000} u_{0}(r)+S_{001} u_{1}(r)\right]=0
\end{aligned}
$$

where $S_{00-1}=S_{001}=0, S_{000}=2$ are calculated from Equation (7).

For the proton-proton system we add the Coulomb effect to Equation (8), $\left[E-V_{c}(r)\right] \rightarrow\left[E-V_{c}(r)+V_{\text {coul }}(r)\right]$.

The numerical techniques necessary to solve equation (8) with this ESC04 potential are explained in chapter 3, Equation (3.28) of [7]. The solutions of $u_{0}$ from Equation (8) are introduced in the $S$ matrix (Equation (10.58) of [7], which is,

$$
S_{l}=\frac{U_{l}\left(r_{n-1}\right) r_{n} h_{l}^{-}\left(k r_{n}\right)-U_{l}\left(r_{n}\right) r_{n-1} h_{l}^{-}\left(k r_{n-1}\right)}{U_{l}\left(r_{n}\right) r_{n-1} h_{l}^{+}\left(k r_{n-1}\right)-U_{l}\left(r_{n-1}\right) r_{n} h_{l}^{+}\left(k r_{n}\right)},
$$

where the $S$ matrix is evaluated in the last two points on a mesh of size $\varepsilon(r=0, \varepsilon, 2 \varepsilon, \cdots N \varepsilon)$. $U_{l}$ are the solutions to Equation (8) with the ESC04 potential previously calculated and $h_{l}$ are the spherical Hankel functions defined in Equation (10.52) of [7].

We insert the numerical solution of the $S$ matrix in the solution of the $S$ matrix for a real potential

$$
S_{l}=e^{2 i \delta_{l}},
$$

where $\delta_{l}$ is real and is known as the phase shift.

Once the $\delta_{0}$ phase shift is found the $a_{n n}$ scattering length and the effective range $r_{n n}$ are calculated. For $l=0$ the expression for $k \cot \left(\delta_{0}\right)$ can be parameterized in the following form,

$$
k \cot \left(\delta_{0}\right)=-\frac{1}{a}+\frac{1}{2} r_{0} k^{2}+\cdots .
$$

The quantity $a$ is called the scattering length and $r_{0}$ is known as the effective range.

In the limit of low energies the scattering length is given in terms of the s-wave phase shift (see appendix B of [8]),

$$
a=\lim _{k \rightarrow 0} \Re\left\{-\frac{1}{k} e^{i \delta_{0}} \sin \left(\delta_{0}\right)\right\},
$$

where $k^{2}=2 \mu E / \hbar^{2}$ is the center-of-mass momentum (the wave number) and $\mathfrak{R}$ indicates the real part.

\subsection{Extended Soft-Core Potential (ESC04)}

An Extended Soft-core potential is calculated consisting of a central, spin-spin, spin-orbital, and a tensor part. The potential of the ESC04 model is generated by one-bosonexchange (OBE), two-meson-exchange (TME) and meson-pair-exchange (MPE); this potential is calculated and explained in [1-4]. In Figure 1 the total ESC04 potential is plotted as a function of the $r$ distance. In Figure 2 we show the central, spin-spin, spin-orbital, and tensor part of this total potential.

The algoritms for the $Y N$ potential are found in [9].

\section{Results}

\section{The $a_{n n}$ Scattering Length}

The $a_{n n}$ scattering length is calculated obtaining a numerical value $a_{n n}=-18.62497 \mathrm{fm}$ and an effective range of $r_{n n}=2.746615 \mathrm{fm}$. We use an ESC04 potential below $350 \mathrm{MeV}$. In Figures 3 and 4 the phase shift $\delta\left({ }^{1} S_{0}\right)$ is plotted for the proton-proton and neutron-proton case.

Table 1 shows the results for the low-energy parameters from the scattering lengths and the effective ranges for neutron-proton, proton-proton and neutron-neutron system using the ESC04 interaction.

\section{Conclusions}

In the present work, we have numerically solved the Schrödinger equation with an ESC04 potential and obtained the nucleon-nucleon scattering lengths. Summarizing our main conclusions:

1) Recent calculations using the ESCO4 interaction for nucleon-nucleon dispersion have been realized [4], and reproduced with the Schrödinger equation.

2) The numerical solution of the radial Schrödinger equation has been realized and has been demonstrated to give a good fit to the nucleon-nucleon data.

3) The scattering lengths $a_{p p}, a_{n p}$ and $a_{n n}$ have been calculated and are consistent with the experimental re-

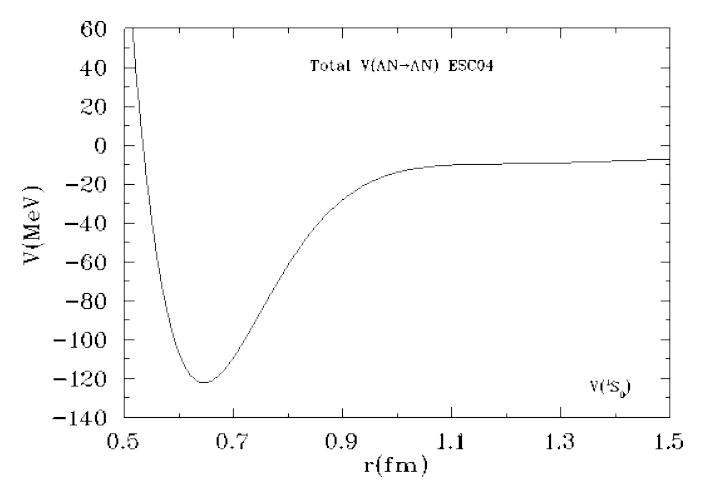

Figure 1. Total potential in the partial wave ${ }^{1} S_{0}$, for $I=1 / 2$.

Table 1. ESC04 low-energy parameters: $S$-wave scattering lengths and effective ranges.

\begin{tabular}{ccc}
\hline & Experimental data & ESC04 \\
\hline$a_{p p}\left({ }^{1} S_{0}\right)$ & $-7.823 \pm 0.010$ & -7.98 \\
$r_{p p}\left({ }^{1} S_{0}\right)$ & $2.794 \pm 0.015$ & 2.762 \\
$a_{n p}\left({ }^{1} S_{0}\right)$ & $-23.715 \pm 0.015$ & -23.801 \\
$r_{n p}\left({ }^{1} S_{0}\right)$ & $2.760 \pm 0.030$ & 2.773 \\
$a_{n n}\left({ }^{1} S_{0}\right)$ & $-18.70 \pm 0.60$ & -18.625 \\
$r_{n n}\left({ }^{1} S_{0}\right)$ & $2.750 \pm 0.11$ & 2.747 \\
\hline
\end{tabular}



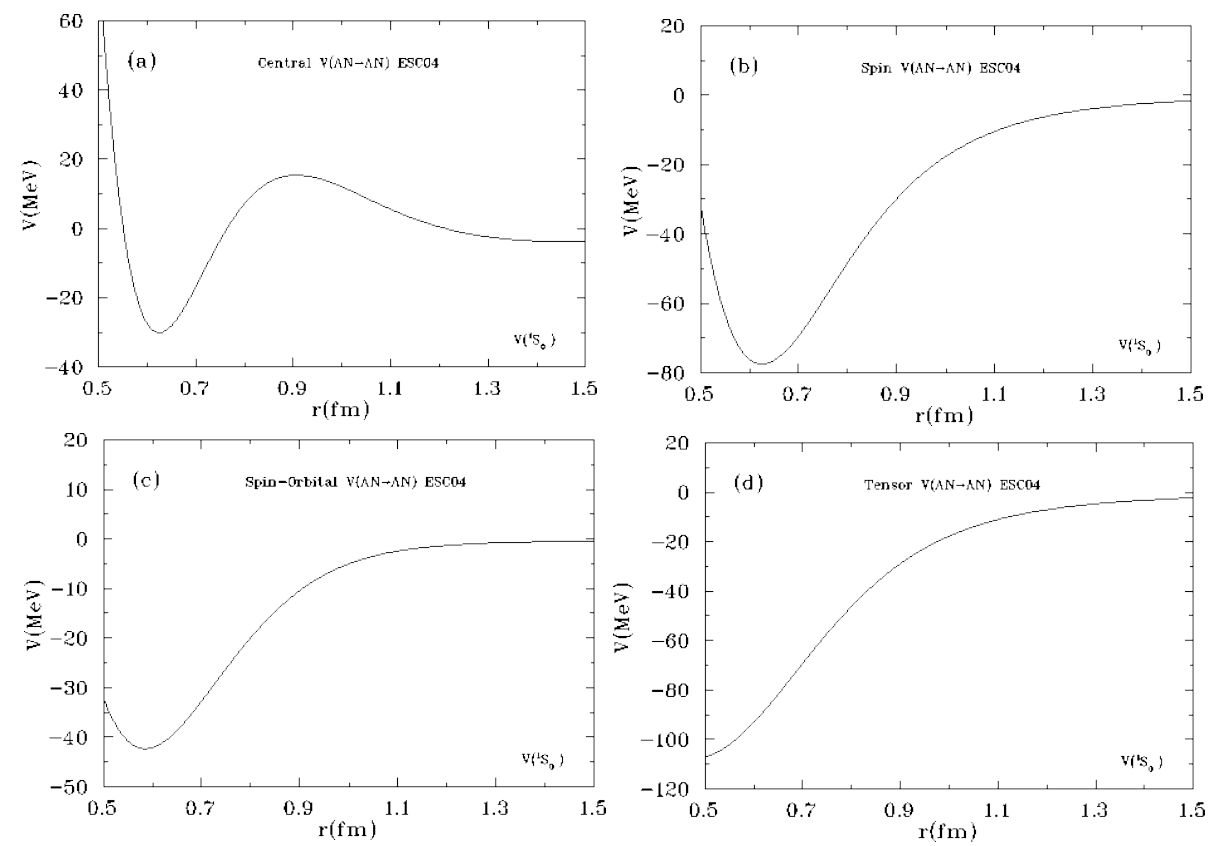

Figure 2. Central (a), spin-spin (b), spin-orbital (c), and tensor (d) part of the YN potential.

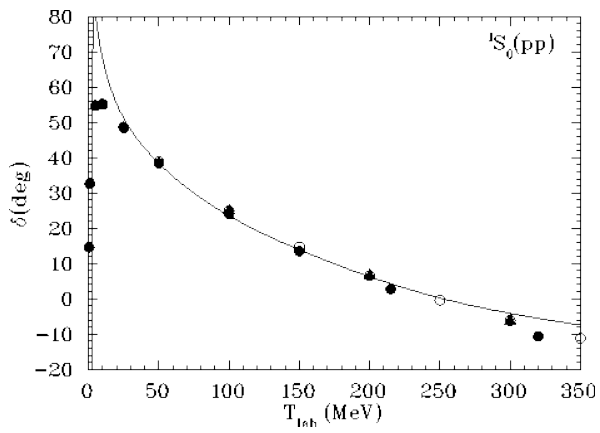

Figure 3. Solid curve, proton-proton $I=1$ phase shifts (degrees), as a function of $T_{l a b}(\mathrm{MeV})$, numerical solution for the ESC04 model. Dots, phases of the Rijken analysis [4]. Circles, s.e. phases of the Nijmegen93 PW analysis. Triangles, the m.e. phases of the Nijmegen93 PW analysis [10].

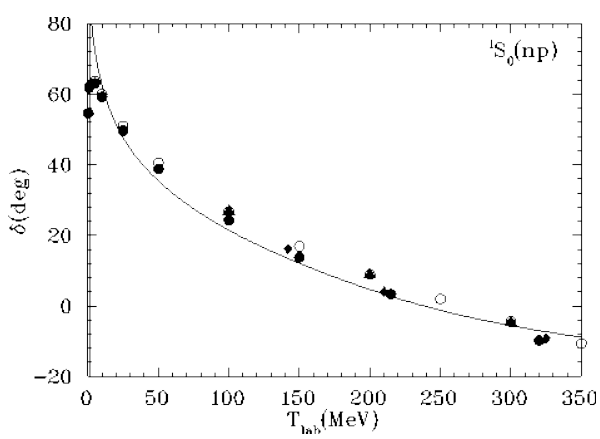

Figure 4. Solid curve, neutron-proton $I=0$ phase shifts (degrees), as a function of $T_{\text {lab }}(\mathrm{MeV})$, numerical solution for the ESC04 model. Dots, phases of the Rijken analysis [4]. Circles, s.e. phases of the Nijmegen93 PW analysis. Triangles, the m.e. phases of the Nijmegen93 PW analysis [10]. Diamonds, Bugg s.e. [11]. sults. The final value for $a_{n n}$ from this study is $a_{n n}=$ $-18.625 \mathrm{fm}$. Results from previous studies are

$$
\begin{aligned}
a_{n n} & =-18.60 \pm 0.34 \pm 0.26 \pm 0.30 \mathrm{fm} \\
& =-18.60 \pm 0.52 \mathrm{fm} \\
a_{n n} & =-18.70 \pm 0.42 \pm 0.39 \pm 0.30 \mathrm{fm} \\
& =-18.70 \pm 0.65 \mathrm{fm}
\end{aligned}
$$

and

$$
\begin{aligned}
a_{n n} & =-18.63 \pm 0.10 \pm 0.44 \pm 0.30 \mathrm{fm} \\
& =-18.63 \pm 0.48 \mathrm{fm}
\end{aligned}
$$

The presented ESC model is thus successful in describing the NN data.

\section{Acknowledgements}

This work was partially supported by PIEIC-UNACH 2012 and SIINV UNACH 2012.

\section{REFERENCES}

[1] Th. A. Rijken and V. G. J. Stocks, "Soft two-Meson-Exchange Nucleon-Nucleon Potentials. I. Planar and CrossedBox Diagrams”, Physical Review C, Vol. 54, No. 6, 1996, pp. 2851-2868.

http://dx.doi.org/10.1103/PhysRevC.54.2851

[2] Th. A. Rijken and V. G. J. Stocks, "Soft Two-MesonExchange Nucleon-Nucleon Potentials. II. One-Pair and Two-Pair Diagrams”, Physical Review C, Vol. 54, No. 6, 1996, pp. 2869-2882.

http://dx.doi.org/10.1103/PhysRevC.54.2869

[3] Th. A. Rijken, H. Polinder and J. Nagata, "Extended- 
Soft-Core NN Potentials in Momentum Space. I. Pseudoscalar-Pseudoscalar Exchange Potentials”, Physical Review C, Vol. 66, No. 4, 2002, pp. 044008-1-044008-19. http://dx.doi.org/10.1103/PhysRevC.66.044008

[4] Th. A. Rijken, "Extended-Soft-Core Baryon-Baryon Model. I. Nucleon-Nucleon Scattering with the ESC04 Interaction,” Physical Review C, Vol. 73, No. 4, 2006, pp. 044007-1-044007-16. http://dx.doi.org/10.1103/PhysRevC.73.044007

[5] J. M. Eisenberg and W. Greiner, "Microscopy Theory of the Nucleus," North-Holland Publishing Company, Amsterdam, 1972.

[6] J. M. Eisenberg and W. Greiner, "Excitation Mechanisms of the Nucleus," North-Holland Publishing Company, Amsterdam, 1972.

[7] W. R. Gibbs, "Computation in Modern Physics," 3rd Edition, World Scientific Publishing, Singapore, 2006.

[8] S. S. M. Wong, "Introductory Nuclear Physics," 2nd Edition, Wiley-VCH Verlag GmbH \& Co. KGaA, New York, 2004.

[9] ESC04 YN Potentials. (2006) http://nn-online.org

[10] V. G. J. Stocks, R. A. M. Klomp M. C. M. Rentmeester and J. J. de Swart, "Partial-Wave Analysis of All Nucleon-Nucleon Scattering Data Below $350 \mathrm{MeV}$,” Physical Review C, Vol. 48, No. 2, 1993, pp. 792-815. http://dx.doi.org/10.1103/PhysRevC.48.792
[11] D. V. Bugg and R. A. Bryan, "Comments on np Elastic Scattering, 142-800 MeV,” Nuclear Physics A, Vol. 540, No. 3-4, 1992, pp. 449-460. http://dx.doi.org/10.1016/0375-9474(92)90168-J

[12] B. Gabioud, J. C. Alder, C. Joseph, J.-F. Loude, N. Morel, A. Perrenoud, J. P. Perroud, M. T. Tran, E. Winkelmann, W. Dahme, H. Panke, D. Renker, C. Zupancic, G. Strassner and P. Truol, "n-n Scattering Length from the Photon Spectra of the Reactions $\pi$-d $\rightarrow \gamma$ nn and $\pi-p \rightarrow \gamma n$," Physical Review Letters, Vol. 42, No. 23, 1979, pp. 1508-1511; http://dx.doi.org/10.1103/PhysRevLett.42.1508

[13] O. Schori, B. Gabioud, C. Joseph, J. P. Perroud, D. Rüegger, M. T. Tran, P. Truöl, E. Winkelmann and W. Dahme, "Measurement of the Neutron-Neutron Scattering Length Ann with the Reaction $\pi$-d $\rightarrow$ nn $\gamma$ in Complete Kinematics,” Physical Review C, Vol. 35, No. 6, 1987, pp. 2252-2257. http://dx.doi.org/10.1103/PhysRevC.35.2252

[14] Q. Chen, C. R. Howell, T. S. Carman, W. R. Gibbs, B. F. Gibson, A. Hussein, M. R. Kiser, G. Mertens, C. F. Moore, C. Morris, A. Obst, E. Pasyuk, C. D. Roper, F. Salinas, H. R. Setze, I. Slaus, S. Sterbenz, W. Tornow, R. L. Walter, C. R. Whiteley and M. Whitton, "Measurement of the Neutron-Neutron Scattering Length Using the $\pi$-d Capture Reaction,” Physical Review C, Vol. 77, No. 5, 2008, pp. 054002-1-054002-19. http://dx.doi.org/10.1103/PhysRevC.77.054002 